\title{
Lower bounds for identifying codes in some infinite grids
}

\author{
Ryan Martin* Brendon Stanton \\ Department of Mathematics \\ Iowa State University \\ Ames, IA 50010
}

Submitted: Apr 20, 2010; Accepted: Aug 27, 2010; Published: Sep 13, 2010

Mathematics Subject Classification: 05C70 (68R10, 94B65)

\begin{abstract}
An $r$-identifying code on a graph $G$ is a set $C \subset V(G)$ such that for every vertex in $V(G)$, the intersection of the radius- $r$ closed neighborhood with $C$ is nonempty and unique. On a finite graph, the density of a code is $|C| /|V(G)|$, which naturally extends to a definition of density in certain infinite graphs which are locally finite. We present new lower bounds for densities of codes for some small values of $r$ in both the square and hexagonal grids.
\end{abstract}

\section{Introduction}

Given a connected, undirected graph $G=(V, E)$, we define $B_{r}(v)$-called the ball of radius $r$ centered at $v$ to be

$$
B_{r}(v)=\{u \in V(G): d(u, v) \leqslant r\} .
$$

We call any nonempty subset $C$ of $V(G)$ a code and its elements codewords. A code $C$ is called $r$-identifying if it has the properties:

1. $B_{r}(v) \cap C \neq \emptyset$

2. $B_{r}(u) \cap C \neq B_{r}(v) \cap C$, for all $u \neq v$

When $C$ is understood, we define $I_{r}(v)=I_{r}(v, C)=B_{r}(v) \cap C$. We call $I_{r}(v)$ the identifying set of $v$.

Vertex identifying codes were introduced in [6] as a way to help with fault diagnosis in multiprocessor computer systems. Codes have been studied in many graphs, but of

\footnotetext{
*Research supported in part by NSA grant H98230-08-1-0015 and NSF grant DMS 0901008 and by an Iowa State University Faculty Professional Development grant.
} 
particular interest are codes in the infinite triangular, square, and hexagonal lattices as well as the square lattice with diagonals (king grid). For each of these graphs, there is a characterization so that the vertex set is $\mathbb{Z} \times \mathbb{Z}$. Let $Q_{m}$ denote the set of vertices $(x, y) \in \mathbb{Z} \times \mathbb{Z}$ with $|x| \leqslant m$ and $|y| \leqslant m$. We may then define the density of a code $C$ by

$$
D(C)=\limsup _{m \rightarrow \infty} \frac{\left|C \cap Q_{m}\right|}{\left|Q_{m}\right|} .
$$

Our first two theorems, Theorem 1 and Theorem 2, rely on a key lemma, Lemma 6, which gives a lower bound for the density of an $r$-identifying code assuming that we are able to show that no codeword appears in "too many" identifying sets of size 2 . Theorem 1 follows immediately from Lemma 6 and Lemma 7 while Theorem 2 follows immediately from Lemma 6 and Lemma 8.

Theorem 1 The minimum density of a 2-identifying code of the hex grid is at least 1/5.

Theorem 2 The minimum density of a 2-identifying code of the square grid is at least $3 / 19 \approx 0.1579$.

Theorem 2 can be improved via Lemma 9, which has a more detailed and technical proof than the prior lemmas. The idea the lemma is that even though it is possible for a codeword to be in 8 identifying sets of size 2, this forces other potentially undesirable things to happen in the code. We use the discharging method to show that on average a codeword can be involved in no more than 7 identifying sets of size 2. Lemma 9 leads to the improvement given in Theorem 2.

Theorem 3 The minimum density of a 2-identifying code of the square grid is at least $6 / 37 \approx 0.1622$.

The paper is organized as follows: Section 2 focuses on some key definitions that we use throughout the paper, provides the proof of Lemma 6 and provides some other basic facts. Section 3 states and proves Lemma 7 from which Theorem 1 immediately follows. It is possible to also use this technique to show that the density of a 3-identifying code is at least $3 / 25$, but the proof is long and the improvement is minor so we will exclude it here. (The proof of this fact will appear in the second author's dissertation [7]). Section 4 gives the proofs of Lemma 8 and 9. Finally, in Section 5, we give some concluding remarks and a summary of known results.

\section{Definitions and General Lemmas}

Let $G_{S}$ denote the square grid. Then $G_{S}$ has vertex set $V\left(G_{S}\right)=\mathbb{Z} \times \mathbb{Z}$ and

$$
E\left(G_{S}\right)=\{\{u, v\}: u-v \in\{(0, \pm 1),( \pm 1,0)\}\}
$$

where subtraction is performed coordinatewise.

THE ELECTRONiC Journal of COMBinatorics 17 (2010), \#R122 
Let $G_{H}$ represent the hex grid. We will use the so-called "brick wall" representation, whence $V\left(G_{H}\right)=\mathbb{Z} \times \mathbb{Z}$ and

$$
E\left(G_{H}\right)=\left\{\{u=(i, j), v\}: u-v \in\left\{\left(0,(-1)^{i+j+1}\right),( \pm 1,0)\right\}\right\} .
$$

Consider an $r$-identifying code $C$ for a graph $G=(V, E)$. Let $c, c^{\prime} \in C$ be distinct. If $I_{r}(v)=\left\{c, c^{\prime}\right\}$ for some $v \in V(G)$ we say that

1. $c^{\prime}$ forms a pair (with $c$ ) and

2. $v$ witnesses a pair (that contains $c$ ).

For $c \in C$, we define the set of witnesses of pairs that contain $c$. Namely,

$$
P(c)=\left\{v: I_{r}(v)=\left\{c, c^{\prime}\right\} \text {, for some } c^{\prime}(\neq c)\right\} .
$$

We also define $p(c)=|P(c)|$. In other words, $P(c)$ is the set of all vertices that witness a pair containing $c$ and $p(c)$ is the number of vertices that witness a pair containing $c$. Furthermore, we call $c$ a $k$-pair codeword if $p(c)=k$.

We start by noting two facts about pairs which are true for any code on any graph.

Fact 4 Let $c$ be a codeword and $S$ be a subset of $P(c)$. If $v \notin S$ and $B_{2}(v) \subset \bigcup_{s \in S} B_{2}(s)$, then $v \notin P(c)$.

Proof. Suppose $v$ witnesses a pair containing $c$. Hence, $I_{2}(v)=\left\{c, c^{\prime}\right\}$ for some $c^{\prime} \neq c$. Then $c^{\prime} \in B_{2}(v)$ and so $c^{\prime} \in B_{2}(s)$ for some $s \in S$. But then $\left\{c, c^{\prime}\right\} \subset I_{2}(s)$. But since $I_{2}(s) \neq I_{2}(v),\left|I_{2}(s)\right|>2$, contradicting the fact that $s$ witnesses a pair. Hence $v$ does not witness such a pair.

Fact 5 Let $c$ be a codeword and $S$ be any set with $|S|=k$. If $v \in S$ and

$$
B_{2}(v) \subset \bigcup_{\substack{s \in S \\ s \neq v}} B_{2}(s)
$$

then at most $k-1$ vertices in $S$ witness pairs containing $c$.

Proof. The result follows immediately from Fact 4 . If each vertex in $S-\{v\}$ witnesses a pair, then $v$ cannot witness a pair. Hence, either $v$ does not witness a pair or some vertex in $S$ does not witness a pair.

Lemma 6 is a general statement about vertex-identifying codes and has a similar proof to Theorem 2 in [6]. In fact, Cohen, Honkala, Lobstein and Zémor [3] use a nearly identical technique to prove lower bounds for 1-identifying codes in the king grid. Their computations can be used to prove a slightly stronger statement that implies Lemma 6 . We will discuss the connection more in Section 5. 
Lemma 6 Let $C$ be an r-identifying code for the square or hex grid. Let $p(c) \leqslant k$ for any codeword. Let $D(C)$ represent the density of $C$, then if $b_{r}=\left|B_{r}(v)\right|$ is the size of a ball of radius $r$ centered at any vertex $v$,

$$
D(C) \geqslant \frac{6}{2 b_{r}+4+k} .
$$

Proof. We first introduce an auxiliary graph $\Gamma$. The vertices of $\Gamma$ are the vertices in $C$ and $c$ is adjacent to $c^{\prime}$ if and only if $c$ forms a pair with $c^{\prime}$. Then we clearly have $\operatorname{deg}_{\Gamma}(c)=p(c)$. Let $\Gamma\left[C \cap Q_{m}\right]$ denote the induced subgraph of $\Gamma$ on $C \cap Q_{m}$. It is clear that if $\operatorname{deg}_{\Gamma}(c) \leqslant k$ then $\operatorname{deg}_{\Gamma\left[C \cap Q_{m}\right]} \leqslant k$.

The total number of edges in $\Gamma\left[C \cap Q_{m}\right]$ by the handshaking lemma is

$$
\frac{1}{2} \sum_{c \in \Gamma\left[C \cap Q_{m}\right]} \operatorname{deg}_{\Gamma\left[C \cap Q_{m}\right]} \leqslant(k / 2)\left|C \cap Q_{m}\right| .
$$

But by our observation above, we note that the total number of pairs in $C \cap Q_{m}$ is equal to the number of edges in $\Gamma\left[C \cap Q_{m}\right]$. Denote this quantity by $P_{m}$. Then

$$
P_{m} \leqslant(k / 2)\left|C \cap Q_{m}\right| .
$$

Next we turn our attention to the grid in question. The arguments work for either the square or hex grid. Note that if $C$ is an $r$-identifying code on the grid, $C \cap Q_{m}$ may not be a valid $r$-identifying code for $Q_{m}$. Hence, it is important to proceed carefully. Fix $m>r$. By definition, $Q_{m-r}$ is a subgraph of $Q_{m}$. Further, for each vertex $v \in V\left(Q_{m-r}\right)$, $B_{r}(v) \subset V\left(Q_{m}\right)$. Hence $C \cap Q_{m}$ must be able to distinguish between each vertex in $Q_{m-r}$.

Let $n=\left|Q_{m}\right|$ and $K=\left|C \cap Q_{m}\right|$. Let $v_{1}, v_{2}, v_{3}, \ldots, v_{n}$ be the vertices of $Q_{m}$ and let $c_{1}, c_{2}, \ldots, c_{K}$ be our codewords. We consider the $n \times K$ binary matrix $\left\{a_{i j}\right\}$ where $a_{i j}=1$ if $c_{j} \in I_{r}\left(v_{i}\right)$ and $a_{i j}=0$ otherwise. We count the number of non-zero elements in two ways.

On the one hand, each column can contain at most $b_{r}$ ones since each codeword occurs in $B_{r}\left(v_{i}\right)$ for at most $b_{r}$ vertices. Thus, the total number of ones is at most $b_{r} \cdot K$.

Counting ones in the other direction, we will only count the number of ones in rows corresponding to vertices in $Q_{m-r}$. There can be at most $K$ of these rows that contain a single one and at most $P_{m}$ of these rows which contain 2 ones. Then there are $\left|Q_{m-k}\right|-$ $K-P_{m}$ left corresponding to vertices in $Q_{m-k}$ and so there must be at least 3 ones in each of these rows. Thus the total number of ones counted this way is at least $K+2 P_{m}+$ $3\left(\left|Q_{m-r}\right|-K-P_{m}\right)=-2 K+3\left|Q_{m-r}\right|-P_{m}$. Thus

$$
b_{r} K \geqslant-2 K+3\left|Q_{m-r}\right|-P_{m} .
$$

But since $P_{m} \leqslant(k / 2) K$, this gives

$$
b_{r} K \geqslant-2 K+3\left|Q_{m-r}\right|-(k / 2) K .
$$


Rearranging the inequality and replacing $K$ with $\left|C \cap Q_{m}\right|$ gives

$$
\frac{\left|C \cap Q_{m}\right|}{\left|Q_{m-r}\right|} \geqslant \frac{6}{2 b_{r}+4+k} .
$$

Then

$$
\begin{aligned}
D(C) & =\limsup _{m \rightarrow \infty} \frac{\left|C \cap Q_{m}\right|}{\left|Q_{m}\right|} \\
& =\limsup _{m \rightarrow \infty} \frac{\left|C \cap Q_{m}\right|}{\left|Q_{m-r}\right|} \cdot \limsup _{m \rightarrow \infty} \frac{\left|Q_{m-r}\right|}{\left|Q_{m}\right|} \\
& \geqslant \frac{6}{2 b_{r}+4+k} \cdot \limsup _{m \rightarrow \infty} \frac{(2(m-r)+1)^{2}}{(2 m+1)^{2}} \\
& =\frac{6}{2 b_{r}+4+k} .
\end{aligned}
$$

\section{Lower Bound for the Hexagonal Grid}

Lemma 7 establishes an upper bound of 6 for the degree of the graph $\Gamma$ formed by an $r$-identifying code in the hex grid, which allows us to prove Theorem 1.

Lemma 7 Let $C$ be a 2-identifying code for the hex grid. For each $c \in C, p(c) \leqslant 6$.

Proof. Let $C$ be an $r$-identifying code and $c \in C$ be an arbitrary codeword. Let $u_{1}, u_{2}$, and $u_{3}$ be the neighbors of $c$ and let $\left\{u_{i 1}, u_{i 2}\right\}=B_{1}\left(u_{i}\right)-\left\{u_{i}, c\right\}$.

Case 1: $\left|I_{2}(c)\right| \geqslant 2$

There exists some $c^{\prime} \in C \cap B_{2}(c)$ with $c^{\prime} \neq c$. Without loss of generality, assume that $c^{\prime} \in\left\{u_{1}, u_{11}, u_{12}\right\}$. Since $I_{2}(c), I_{2}\left(u_{1}\right), I_{2}\left(u_{11}\right), I_{2}\left(u_{12}\right) \supseteq\left\{c, c^{\prime}\right\}$ at most one of $c, u_{1}, u_{11}, u_{12}$ witnesses a pair containing $c$.

Now, $p(c) \leqslant 6$ unless each of $u_{2}, u_{3}, u_{21}, u_{22}, u_{31}, u_{32}$ witnesses a pair.

If $u_{2}$ and $u_{3}$ each witness a pair, then we have $u_{i} \notin C$ for $i=1,2,3$; otherwise $I_{2}\left(u_{2}\right)=\left\{c, u_{i}\right\}=I_{2}\left(u_{3}\right)$ and so $u_{2}$ and $u_{3}$ are not distinguishable by our code. Thus, there must be some $c^{\prime \prime} \in C \cap\left(B_{2}\left(u_{2}\right)-\left\{c, u_{1}, u_{2}, u_{3}\right\}\right)$. This forces $c^{\prime \prime} \in B_{2}\left(u_{21}\right) \cup B_{2}\left(u_{22}\right)$ and so either $\left\{c, c^{\prime \prime}\right\} \subseteq I_{2}\left(u_{21}\right)$ or $\left\{c, c^{\prime \prime}\right\} \subseteq I_{2}\left(u_{22}\right)$. Hence, one of these cannot witness a pair and still be distinguishable from $u_{2}$. This ends case 1 .

Case 2: $I_{2}(c)=\{c\}$

First note that $c$ itself does not witness a pair.

If $u_{1}$ witnesses a pair, then there is some $c^{\prime \prime} \in C \cap\left(B_{2}\left(u_{1}\right)-B_{2}(c)\right) \subseteq C \cap\left(B_{2}\left(u_{11}\right) \cup\right.$ $\left.B_{2}\left(u_{12}\right)\right)$ and so either $\left\{c, c^{\prime \prime}\right\} \subseteq I_{2}\left(u_{11}\right)$ or $\left\{c, c^{\prime \prime}\right\} \subseteq I_{2}\left(u_{12}\right)$ and so one of these cannot witness a pair and still be distinguishable from $u_{1}$. Hence at most two of $\left\{u_{1}, u_{11}, u_{12}\right\}$ can witness a pair. 
Likewise at most at most two of $\left\{u_{2}, u_{21}, u_{22}\right\}$ and $\left\{u_{3}, u_{31}, u_{32}\right\}$ can witness a pair. Thus $p(c) \leqslant 6$. This ends both case 2 and the proof of the lemma.

Proof of Theorem 1. Using Lemmas 6 and 7, if $C$ is a 2-identifying code in the hexagonal grid, then

$$
D(C) \geqslant \frac{6}{2 b_{2}+4+6}=\frac{6}{30}=\frac{1}{5} .
$$

\section{Lower Bounds for the Square Grid}

Lemma 8 establishes an upper bound of 8 for the degree of the graph $\Gamma$ formed by an $r$-identifying code in the square grid, which allows us to prove Theorem 2 . Then we prove Lemma 9, which bounds the average degree of $\Gamma$ by 7 , allowing for the improvement in Theorem 3.

It is worth noting that the proof of Lemma 8 could be shortened significantly, but the proof is needed in order to prove Lemma 9, which gives the result in Theorem 3.

Lemma 8 Let $C$ be a 2-identifying code for the square grid. For each $c \in C, p(c) \leqslant 8$.

Proof. Let $c \in C$, a 2-identifying code in the square grid. Without loss of generality, we will assume that $c=(0,0)$.

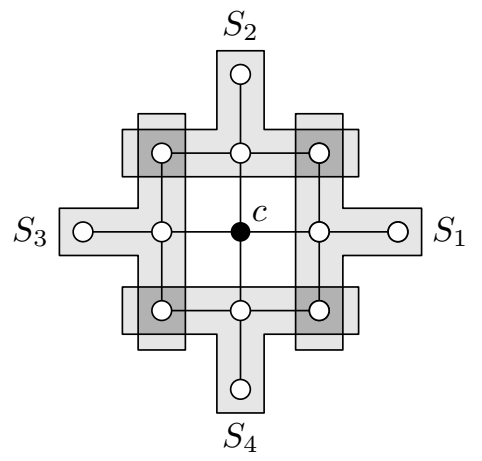

Figure 1: The sets $S_{1}, S_{2}, S_{3}$ and $S_{4}$.

Case 1: $c$ witnesses a pair.

This case implies immediately that $\left|I_{2}(c)\right|=2$. The other codeword in $I_{2}(c)$, namely $c^{\prime}$, is in one of the following 4 sets, the union of which is $B_{2}(c)-\{c\}$. See Figure 1 .

$$
\begin{aligned}
& S_{1}:=\{\quad(1,0), \quad(1,1), \quad(1,-1), \quad(2,0)\} \\
& S_{2}:=\{\quad(0,1), \quad(1,1), \quad(-1,1), \quad(0,2)\} \\
& S_{3}:=\{(-1,0), \quad(-1,1), \quad(-1,-1), \quad(-2,0)\} \\
& S_{4}:=\{(0,-1),(1,-1), \quad(-1,-1),(0,-2)\}
\end{aligned}
$$




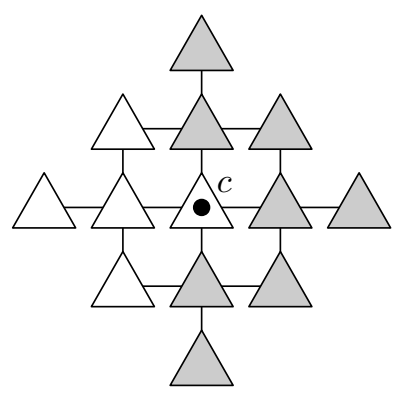

Figure 2: The ball of radius 2 around $c$. A configuration of 9 vertices witnessing pairs is not possible if $\left|I_{2}(c)\right|=2$.

- At most 7 of the vertices in gray triangles may witness a pair.

- At most one of the vertices in white triangles may witness a pair.

If, however, $c^{\prime} \in S_{i}$, then no $s \in S_{i}$ can witness a pair because $\left\{c, c^{\prime}\right\} \subseteq I_{2}(s)$ and $s$ could not be distinguished from $c$. Without loss of generality, assume that $c^{\prime} \in S_{3}$. Thus, all vertices witnessing pairs in $I_{2}(c)$ are in the set

$$
R:=\left\{(x, y):(x, y) \in B_{2}(c), x \geqslant 0\right\} .
$$

But because

$$
B_{2}((1,0)) \subseteq \bigcup_{s \in S_{1} \cup\{c\}} B_{2}(s),
$$

Fact 4 gives that not all members of $S_{1} \cup\{c\}$ can witness a pair. See Figure 2 .

Therefore, $p(c) \leqslant 8$ and, without loss of generality, $c^{\prime} \in S_{3}$ and at least one element of $S_{1}$ does not witness a pair. This ends Case 1 .

Case 2: $c$ does not witness a pair.

This case implies immediately that either $\left|I_{2}(c)\right| \geqslant 3$ or $I_{2}(c)=\{c\}$.

First suppose $\left|I_{2}(c)\right| \geqslant 3$. There must be two distinct codewords $c^{\prime}, c^{\prime \prime} \in S_{1} \cup S_{2} \cup S_{3} \cup S_{4}$. If $c^{\prime}, c^{\prime \prime}$ are in the same set $S_{i}$ for some $i$, then $\left\{c, c^{\prime}, c^{\prime \prime}\right\} \subset I_{2}(s)$ for any $s \in S_{i}$ and so no vertex in $S_{i}$ witnesses a pair. Thus, the only vertices which can witness a pair are in $B_{2}(c)-\left(S_{i} \cup\{c\}\right)$. There are only 7 of these, so $p(c) \leqslant 7$. (See the gray vertices in Figure 2).

If $c^{\prime} \in S_{i}$ and $c^{\prime \prime} \in S_{j}$ for some $i \neq j$, then only one vertex in each of $S_{i}$ and $S_{j}$ can witness a pair. There are at most 5 other vertices not in $S_{i} \cup S_{j}-\{c\}$ and so $p(c) \leqslant 7$.

Thus, if $\left|I_{2}(c)\right| \geqslant 3$, then $p(c) \leqslant 7$.

Second, suppose $I_{2}(c)=\{c\}$. We will define a right angle of witnesses to be subsets of 3 vertices of $I_{2}(c)$ that all witness pairs and are one of the following 8 sets: $\{(1,0),(2,0),(1, \pm 1)\}, \quad\{(0,1),(0,2),( \pm 1,1)\}, \quad\{(-1,0),(-2,0),(-1, \pm 1)\}, \quad$ and $\{(0,-1),(0,-2),( \pm 1,-1)\}$. If a right angle is present then, without loss of generality, let it be $\{(0,1),(0,2),(1,1)\}$. See Figure 3. In order for these all to be witnesses, then $I_{2}((0,1))$ must have one codeword not in $B_{2}((0,2)) \cup B_{2}((1,1))$, which can only be $(-2,1)$. Since $\{(0,0),(-2,1)\} \subseteq B_{2}((-1,1)), B_{2}((-1,0)), B_{2}((-2,0))$, none of those three vertices can witness a pair. 


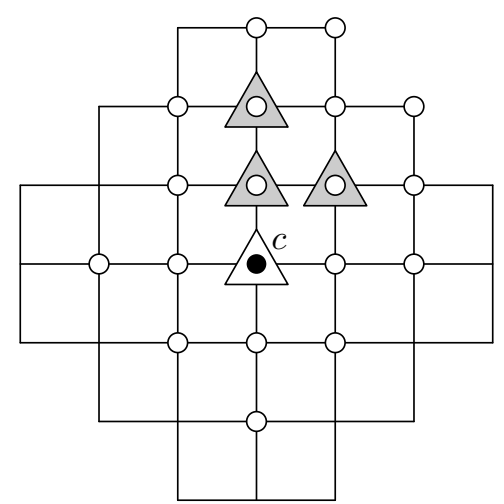

Figure 3: A right angle of witnesses.

- Black circles indicate codewords.

- White circles indicate non-codewords.

- Gray triangles indicate vertices that witness a pair.

- White triangles indicate vertices that do not witness a pair.

No vertices in $B_{2}(c)-\{c\}$ can be codewords, neither can those which are distance no more than 2 from two vertices in this right angle of witnesses.

In addition, $I_{2}((1,1))$ must contain a codeword not in $B_{2}((0,1)) \cup B_{2}((0,2))$, which can only be $(3,1)$. See Figure 4 . Since $\{(0,0),(3,1)\} \subseteq B_{2}((2,0))$, the vertex $(2,0)$ cannot witness a pair.

Finally, it is not possible for all of $(-1,-1),(0,-1),(1,-1),(0,-2)$ to be witnesses because the only member of $B_{2}((0,-1))$ that is not in the union of the second neighborhoods of the others is the vertex $(0,1)$, which cannot be a codeword in this case. Hence, at most 7 members of $B_{2}(c)$ can witness a pair if $B_{2}(c)$ has a right angle of witnesses.

Consequently, if $c$ does not witness a pair and $p(c) \geqslant 8$, then $I_{2}(c)=\{c\}$ and $B_{2}(c)$ fails to have a right angle of witnesses. We can enumerate the remaining possibilities according to how many of the vertices $\{(1,1),(-1,1),(-1,-1),(1,-1)\}$ are witnesses. If 1,2 or 3 of them are witnesses and there is no right angle of witnesses, it is easy to see that there are at most 7 witnesses in $B_{2}(c)$ and so $p(c) \leqslant 7$.

The first remaining case is if 0 of them are witnesses, implying each of the eight vertices $( \pm 1,0),( \pm 2,0),(0, \pm 1)$ and $(0, \pm 2)$ are witnesses. The second remaining case is if 4 of them are witnesses. This implies that at most one of $\{(1,0),(2,0)\}$ are witnesses and similarly for $\{(0,1),(0,2)\},\{(-1,0),(-2,0)\}$ and $\{(0,-1),(0,-2)\}$.

This ends both Case 2 and the proof of the lemma. So, $p(c) \leqslant 8$ with equality only if one of two cases in the previous paragraph holds.

Proof of Theorem 2. Using Lemmas 6 and 8, if $C$ is a 2-identifying code in the square grid, then

$$
D(C) \geqslant \frac{6}{2 b_{2}+4+8}=\frac{6}{38}=\frac{3}{19} .
$$




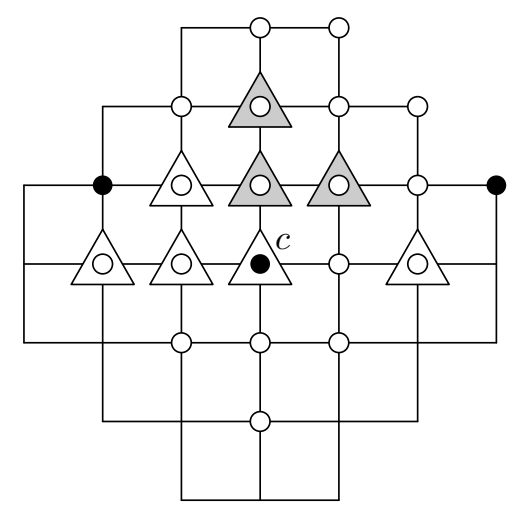

Figure 4: A right angle of witnesses, continuing from Figure 3. Let $c=(0,0)$. Vertices $(-2,1)$ and $(3,1)$ must be codewords and so none of $\{(-1,1),(-1,0),(-2,0),(2,0)\}$ can witness pairs.

Lemma 9 Let $C$ be an r-identifying code for the square grid. Then $\sum_{c \in C \cap Q_{m}} p(c) \leqslant$ $7\left|C \cap Q_{m}\right|$.

Proof. Define

$$
R(c)=\left\{c^{\prime}: I_{2}(v)=\left\{c, c^{\prime}\right\} \text { for some } v \in V\left(G_{S}\right)\right\} .
$$

Suppose that $p(c)=8$ for some $c \in C$. We claim that one of the two following properties holds.

(P1) There exist distinct $c_{1}, c_{2}, c_{3} \in R(c)$ such that $p\left(c_{1}\right) \leqslant 4$ and $p\left(c_{i}\right) \leqslant 6$ for $i=2,3$.

(P2) There exist distinct $c_{1}, c_{2}, c_{3}, c_{4}, c_{5}, c_{6} \in R(c)$ such that $p\left(c_{i}\right) \leqslant 6$ for all $i$.

We will prove this by characterizing all possible 8-pair vertices, but first we wish to define 3 different types of codewords. The definition of each type extends by taking translations and rotations. So, we may assume in defining the types that $c=(0,0)$.

We say that $c$ is a type 1 codeword if $(0,1),(0,-1) \in C$. See Figure 5 .

We say that $c$ is a type 2 codeword if $(-1,2),(2,-1) \in C$. See Figure 6 .

We say that $c$ is a type 3 codeword if $(-2,1),(2,1) \in C$. See Figure 7 .

Claim 10 shows that adjacent codewords do not need to be considered because they are in few pairs.

Claim 10 If $c$ is adjacent to another codeword, then $p(c) \leqslant 6$.

Proof. Without loss of generality, assume that $c=(0,0)$ and that $(0,1)$ is a codeword. Then

$$
(-1,0),(0,0),(0,1),(0,2),(1,0),(1,1),(-1,0),(-1,1)
$$

are all at most distance 2 from both codewords and so at most 1 of them can witness a pair. Thus, the other 7 do not witness pairs containing $c$. Since $\left|B_{2}(c)\right|=13, p(c) \leqslant 13-7=6$. This proves Claim 10.

Claims 11, 12 and 13 show that types 1, 2 and 3 codewords, respectively, are not in many pairs. 
Claim 11 If $c$ is a type 1 codeword, then $p(c) \leqslant 4$.

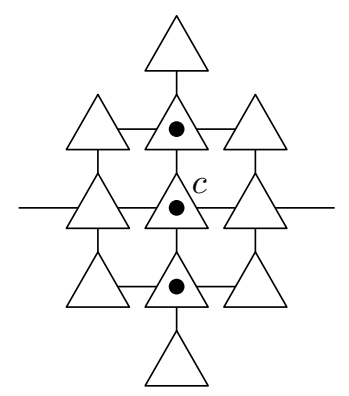

Figure 5: Vertex $c$ is a type 1 codeword. At most 2 of the 11 vertices marked by triangles can witness a pair.

Proof. Without loss of generality, let $c=(0,0)$. We consider all vertices which are distance 2 from $c$ and either $(0,1)$ or $(0,-1)$. There are 11 such vertices and at most 2 of them can witness pairs, so $p(c) \leqslant 4$. See Figure 5 . This proves Claim 11.

Claim 12 If $c$ is a type 2 codeword, then $p(c) \leqslant 6$.

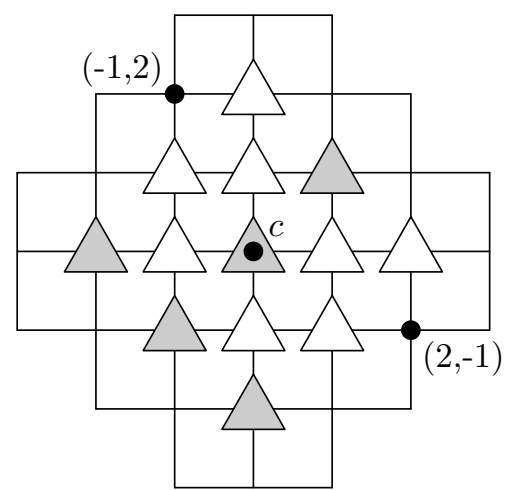

Figure 6: Vertex $c=(0,0)$ is a type 2 codeword. At most 2 of the 8 vertices marked by white triangles can witness pairs. At most 4 of the 5 vertices marked by gray triangles can witness pairs.

Proof. Without loss of generality, let $c=(0,0)$. We consider all vertices which are distance at most 2 from $c$ and distance at most 2 from either $(-1,2)$ or $(2,-1)$. There are 8 such vertices and at most 2 of them can witness pairs. The remaining 5 vertices are $c$ and the vertices in the set $S=\{(-2,0),(-1,-1),(0,-2),(1,1)\}$. But then $B_{2}(c) \subset$ $\bigcup_{s \in S} B_{2}(s)$ and, by Fact 4 at most 4 of those remaining 5 vertices can witness pairs. Thus, $p(c) \leqslant 6$. See Figure 6. This proves Claim 12 .

Claim 13 If $c$ is a type 3 codeword, then $p(c) \leqslant 6$. 


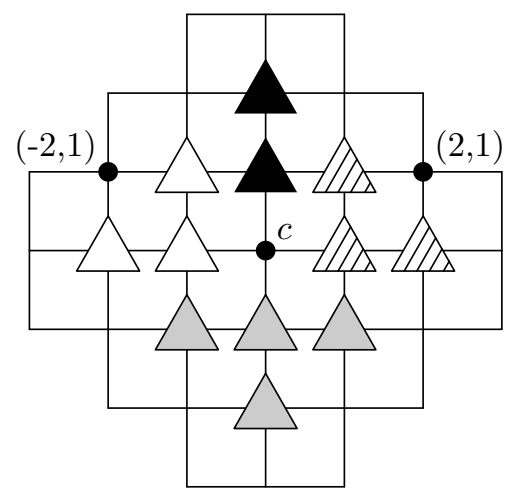

Figure 7: Vertex $c=(0,0)$ is a type 3 codeword.

- $T_{0}$ vertices are black.

- $T_{1}$ vertices are white.

- $T_{2}$ vertices are marked by diagonal lines.

- $T_{3}$ vertices are gray.

Proof. Without loss of generality, let $c=(0,0)$. We partition $B_{2}(c)-\{c\}$ into 4 sets:

$$
\begin{aligned}
& T_{0}:=\{ \\
& (0,1), \quad(0,2)\} \\
& T_{1}:=\{ \\
& (-2,0), \quad(-1,0), \quad(-1,1)\} \\
& T_{2}:=\{ \\
& (2,0), \quad(1,0), \quad(1,1)\} \\
& T_{3}:=\{(-1,-1),(0,-1),(1,-1),(0,-2)\}
\end{aligned}
$$

At most 1 vertex in $T_{0}$ witnesses a pair since $\left|I_{2}(0,1)\right| \geqslant 3$.

At most 1 vertex in $T_{1}$ can witness a pair since every vertex in $T_{1}$ is at most distance 2 from $(-2,1)$. Likewise, at most 1 vertex in $T_{2}$ can witness a pair.

If all vertices in $T_{3}$ witness pairs, then $I_{2}((0,-1))=\{(0,0),(0,1)\}$ since $(0,1)$ is the only vertex in $B_{2}((0,-1))$ which is not in $B_{2}(s)$ for any other $s \in T_{3}$. But then $c$ is adjacent to another codeword, and by Claim $10, p(c) \leqslant 6$. So we may assume that at most 3 vertices in $T_{3}$ form pairs with $c$.

Now, if $c$ does not itself witness a pair, these partitions give $p(c) \leqslant 6$. If $c$ does witness a pair, then there must be another codeword $c^{\prime} \in S_{i}$ for some $i$. But then we see that no other vertex in $S_{i}$ can witness a pair, since every vertex in $S_{i}$ is at most distance two from $c^{\prime}$. Thus, $p(c) \leqslant 6$. See Figure 7. This proves Claim 13 .

We are now ready to characterize the 8-pair codewords.

Claim 14 If $c \in C$ witnesses a pair and $p(c)=8$, then $c$ satisfies property (P1).

Proof. Without loss of generality, let $c=(0,0)$. Recall Case 1 of the proof of Lemma 8 . That is, $p(c) \leqslant 8$ and, without loss of generality, equality implies that there is a $c^{\prime} \in C \cap S_{3}$ and at least one of $S_{1}=\{(1,-1),(1,0),(1,1),(2,0)\}$ does not witness a pair.

If $p(c) \leqslant 7$, the proof is finished, so let us assume that $p(c)=8$ and hence exactly one of the vertices in $S_{1}$ does not witness a pair. We will show that it is $(2,0)$. So, suppose that $(1, y)$ does not witness a pair. Recall that $R=\left\{(x, y):(x, y) \in B_{2}(c), x \geqslant 0\right\}$. 


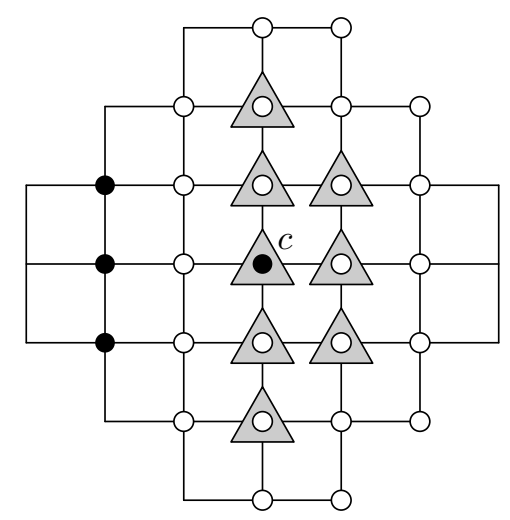

Figure 8: Codeword $c=(0,0)$ witnesses a pair and is an 8-pair codeword. The gray triangles are vertices that form pairs with $c$. Vertex $(-2,0)$ is a type 1 codeword.

If $y \in\{-1,1\}$, then

$$
B_{2}((1,0)) \subseteq \bigcup_{s \in R-\{(1, y),(1,0)\}} B_{2}(s)
$$

and, by Fact 4 , neither $(1, y)$ nor $(1,0)$ witnesses a pair and $p(c) \leqslant 7$.

If $y=0$, then

$$
B_{2}((1,1)) \subseteq \bigcup_{s \in R-\{(1,0),(1,1)\}} B_{2}(s)
$$

and, by Fact 4 , neither $(1,0)$ nor $(1,1)$ witnesses a pair and $p(c) \leqslant 7$. It follows that each vertex in $R^{\prime}=R-\{(2,0)\}$ must witness a pair containing $c$.

Each vertex which is distance 2 or less from 2 vertices in $R^{\prime}$ cannot be a codeword. Thus, $(-2,0)$ is the only vertex in $B_{2}(c)$ other than $c$ which has not been marked as a noncodeword and so $(-2,0) \in C$. Since $(0,0) \in C$, the vertex $(-2,1)$ is the only possibility for a second codeword for $(0,1)$ and $(-2,-1)$ is the only possibility for a second codeword for $(0,-1)$. See Figure 8 .

Then $(-2,0)$ is a type 1 codeword and so it is in at most 4 pairs. Codewords $(-2,1)$ and $(-2,-1)$ are both adjacent to another codeword, so they are in at most 6 pairs. Hence, $c$ satisfies Property (P1). This proves Claim 14.

Claim 15 If $c \in C$ does not witness a pair and $p(c)=8$, then $c$ satisfies either property (P1) or property (P2).

Proof. Without loss of generality, let $c=(0,0)$. Recall Case 2 of the proof of Lemma 8 . That is, $p(c) \leqslant 8$ and, without loss of generality, equality implies $I_{2}(c)=\{c\}$. Furthermore, one of the following two cases occurs:

(1) The eight witnesses are the vertices $( \pm 1,0),( \pm 2,0),(0, \pm 1)$ and $(0, \pm 2)$.

(2) The witnesses include $\{(1,1),(-1,1),(-1,-1),(1,-1)\}$ as well as exactly one of each of the following pairs: $\{(1,0),(2,0)\},\{(0,1),(0,2)\},\{(-1,0),(-2,0)\}$ and $\{(0,-1),(0,-2)\}$. 
If case (1) occurs, then the eight witnesses are the vertices $( \pm 1,0),( \pm 2,0),(0, \pm 1)$ and $(0, \pm 2)$. In this case, simply observe that $B_{2}((1,0))$ is a subset of the other seven witnesses. This contradicts Fact 5 and so this case cannot occur.

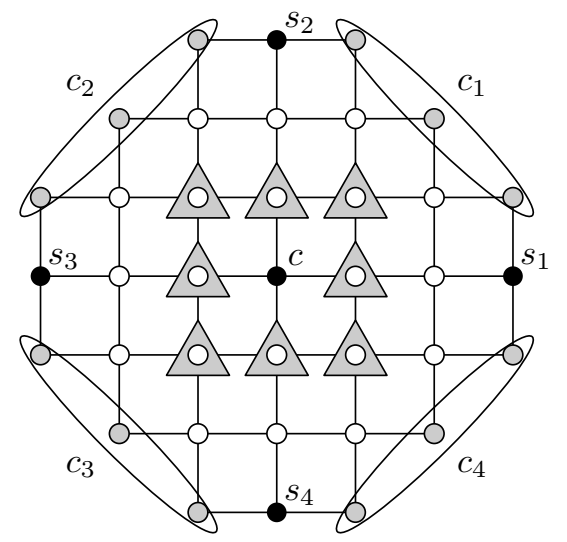

Figure 9: Codeword $c=(0,0)$ fails to witness a pair and is an 8-pair codeword. Exactly one of the gray vertices in each oval is a codeword.

So, we may assume that case $(2)$ occurs. The vertex $(2,1)$ cannot be a codeword because $\{(0,0),(2,1)\} \subseteq B_{2}((1,1)), B_{2}((1,0)), B_{2}((2,0))$ and so at most one of these three vertices witness pairs, a contradiction to case (2). By symmetry, none of the following vertices are codewords:

$$
(2,1),(1,2),(-1,2),(-2,1),(-2,-1),(-1,-2),(1,-2),(2,-1) .
$$

In order to distinguish $(1,0)$ from $(0,0)$, the only vertex available to be a codeword is $s_{1}:=(3,0)$ and symmetrically, $s_{2}:=(0,3), s_{3}:=(-3,0)$ and $s_{4}:=(0,-3)$ are codewords. This implies that each of $(1,0),(0,1),(-1,0)$ and $(0,-1)$ witness pairs.

Then, for the other 4 pairs, there are exactly 3 choices for codewords which are not in the ball of radius 2 for any of our other pairs. See Figure 9.

\begin{tabular}{c|ccl} 
Vertex & \multicolumn{3}{|c}{ Other Codeword } \\
\hline$(1,1)$ & $c_{1} \in$ & $\{(3,1),(2,2),(1,3)\}$ \\
$(-1,1)$ & $c_{2}$ & $\in$ & $\{(-1,3),(-2,2),(-3,1)\}$ \\
$(-1,-1)$ & $c_{3} \in$ & $\in(-3,-1),(-2,-2),(-1,-3)\}$ \\
$(1,-1)$ & $c_{4} \in\{(1,-3),(2,-2),(3,-1)\}$
\end{tabular}

For each $c_{i}$, either $c_{i}$ is adjacent to another codeword or $c_{i}$ is a type 2 codeword. Claims 10 and 12 imply that, in either case, $p\left(c_{i}\right) \leqslant 6$. It remains to show that one of the following holds: (1) There exist $i \neq j$ such that $p\left(s_{i}\right) \leqslant 6$ and $p\left(s_{j}\right) \leqslant 6$, hence $c$ satisfies (P2). (2) There exists an $i$ such that $p\left(s_{i}\right) \leqslant 4$, hence $c$ satisfies (P1).

First, suppose that there are $c_{i}, c_{j}, i \neq j$ such that $c_{i}$ is adjacent to $s_{k}$ and $c_{j}$ is adjacent to $s_{\ell}$. If $k=\ell$, then $s_{k}$ is a type 1 codeword and so $p\left(s_{k}\right) \leqslant 4$. If $k \neq \ell$, then both $s_{k}$ and $s_{\ell}$ are adjacent to another codeword and so $p\left(s_{k}\right) \leqslant 6$ and $p\left(s_{\ell}\right) \leqslant 6$. Either (P1) or (P2) is satisfied, respectively. 
If there is at most one $c_{i}$ such that $c_{i}$ is adjacent to $s_{k}$ for some $k$, then we have three codewords of the form $( \pm 2, \pm 2)$. Without loss of generality, assume that $(2,2),(2,-2)$, and $(-2,2)$ are codewords. In this case, $(3,0)$ and $(0,3)$ are type 3 codewords and hence $p((3,0)) \leqslant 6$ and $p((0,3)) \leqslant 6$. So again, $(\mathrm{P} 2)$ is satisfied.

This proves Claim 15.

Finally, we can finish the proof of Lemma 9 by way of the discharging method. (For a more extensive application of the discharging method on vertex identifying codes, see Cranston and $\mathrm{Yu}[4]$.) Let $\Gamma$ denote an auxiliary graph with vertex set $C \cap Q_{m}$ for some $m$. There is an edge between two vertices $c$ and $c^{\prime}$ if and only if $I_{2}(v)=\left\{c, c^{\prime}\right\}$ for some $v \in V\left(G_{S}\right)$. For each vertex $v$ in our auxiliary graph $\Gamma$, we assign it an initial charge of $d(v)-7$. Note that $\sum_{c \in C \cap Q_{m}} p(c)-7=\sum_{v \in \Gamma} \operatorname{deg}_{\Gamma}(v)-7$. We apply the following discharging rules if $\operatorname{deg}_{\Gamma}(v)=8$.

1. If $v$ is adjacent to one vertex of degree at most 4 and two of degree at most 6 (condition $(\mathrm{P} 1)$ ), then discharge $2 / 3$ to a vertex of degree at most 4 and $1 / 6$ to two vertices of degree at most 6 .

2. If $v$ is adjacent to 6 vertices of degree at most 6 (condition (P2)), then discharge $1 / 6$ to 6 neighbors of degree at most 6 .

We have proven that one of the above cases is possible. Let $e(v)$ be the charge of each vertex after discharging takes place. We show that $e(v) \leqslant 0$ for each vertex in $\Gamma$.

If $\operatorname{deg}_{\Gamma}(v)=8$, then our initial charge was 1 . In either of the two cases, we are discharging a total of 1 unit to its neighbors. Since no degree 8 vertex receives a charge from any other vertex, we have $e(v)=0$.

If $d(v)=7$ then its initial charge is 0 and it neither gives nor receives a charge and so $e(v)=0$.

If $5 \leqslant \operatorname{deg}_{\Gamma}(v) \leqslant 6$, then its initial charge was at most -1 . Since this vertex has at most 6 neighbors and can receive a charge of at most 1/6 from each of them, this gives $e(v) \leqslant 0$.

If $\operatorname{deg}_{\Gamma}(v) \leqslant 4$, then its initial charge was at most -3 . Since this vertex has at most 3 neighbors and can receive a charge of at most $2 / 3$ from each of them, this gives $e(v) \leqslant-1 / 3<0$.

Since no vertex can have degree more than 8 , this covers all of the cases. Then we have

$$
\sum_{c \in C \cap Q_{m}}(p(c)-7)=\sum_{v \in \Gamma}\left(\operatorname{deg}_{\Gamma}(v)-7\right)=\sum_{v \in \Gamma} e(v) \leqslant 0 .
$$

Therefore, it follows that $\sum_{c \in C \cap Q_{m}} p(c) \leqslant \sum_{c \in C \cap Q_{m}} 7=7\left|C \cap Q_{m}\right|$.

Proof of Theorem 3. Consider $Q_{m}$ and let $C$ be an $r$-identifying code for $G_{S}$ and $C \cap Q_{m}=\left\{c_{1}, c_{2}, \ldots, c_{K}\right\}$. Recall inequality (1) from Theorem 6. In this case, $b_{2}=13$ and Lemma 9 shows that

$$
P_{m} \leqslant \frac{1}{2} \sum_{c \in C \cap Q_{m}} p(c) \leqslant \frac{7}{2}\left|C \cap Q_{m}\right| .
$$


Substituting the above inequality into inequality (1) and rearranging gives

$$
\frac{\left|C \cap Q_{m}\right|}{\left|Q_{m-r}\right|} \geqslant \frac{6}{37} .
$$

Taking the limit as $m \rightarrow \infty$ gives the desired $D(C) \geqslant 6 / 37$, completing the proof.

\section{Conclusions}

The technique used for Lemma 6 is similar to the one in Cohen, Honkala, Lobstein and Zémor [3]. Define

$$
\ell=\min _{c \in C}\left|\left\{v \in B_{r}(c):\left|I_{r}(v)\right| \geqslant 3\right\}\right| .
$$

An anonymous referee points out that the computations in [3] can lead one to conclude that

$$
D(C) \geqslant \frac{6}{3 b_{r}+3-\ell}
$$

From our definitions

$$
k=\max _{c \in C}\left|\left\{v \in B_{r}(c):\left|I_{r}(v)\right|=2\right\}\right| .
$$

Since $k+\ell \geqslant b_{r}-1$, one can use $(2)$ to derive the result in Lemma 6 .

As the referee also points out, $k+\ell \leqslant b_{r}$, so the denominator could potentially be improved by an additive factor of 1 if it were possible to show that $k+\ell=b_{r}$.

Below is a table noting our improvements.

\begin{tabular}{|c|c||c|c|}
\hline \multicolumn{4}{|c|}{ Hex Grid } \\
\hline$r$ & previous lower bounds & new lower bounds & upper bounds \\
\hline 2 & $2 / 11 \approx 0.1818^{[6]}$ & $1 / 5=0.2$ & $4 / 19 \approx 0.2105^{[2]}$ \\
\hline 3 & $2 / 17 \approx 0.1176^{[1]}$ & $3 / 25=0.12^{[7]}$ & $1 / 6 \approx 0.1667^{[2]}$ \\
\hline \multicolumn{4}{|c|}{ Square Grid } \\
\hline 2 & $3 / 20=0.15^{[1]}$ & $6 / 37 \approx 0.1622$ & $5 / 29 \approx 0.1724^{[5]}$ \\
\hline
\end{tabular}

This technique works quite well for small values of $r$, but we note that $b_{r}=\left|B_{r}(v)\right|$ grows quadratically in $r$, so the denominator in Lemma 6 would grow quadratically. But the known the lower bounds for $r$-identifying codes is proportional to $1 / r$ in all of the well-studied grids (square, hexagonal, triangular and king). Therefore, the technique is less effective as $r$ grows.

\section{Acknowledgements}

We would like to thank an anonymous referee for making helpful suggestions and directing us to the paper [3]. 


\section{References}

[1] Irène Charon, Iiro Honkala, Olivier Hudry, and Antoine Lobstein. General bounds for identifying codes in some infinite regular graphs. Electron. J. Combin., 8(1):R39, 2001.

[2] Irène Charon, Olivier Hudry, and Antoine Lobstein. Identifying codes with small radius in some infinite regular graphs. Electron. J. Combin., 9(1):R11, 2002.

[3] Gérard D. Cohen, Iiro Honkala, Antoine Lobstein, and Gilles Zémor. On codes identifying vertices in the two-dimensional square lattice with diagonals. IEEE Trans. Comput., 50(2):174-176, 2001.

[4] Daniel W. Cranston and Gexin Yu. A new lower bound on the density of vertex identifying codes for the infinite hexagonal grid. Electron. J. Combin., 16(1):R113, 2009.

[5] Iiro Honkala and Antoine Lobstein. On the density of identifying codes in the square lattice. J. Combin. Theory Ser. B, 85(2):297-306, 2002.

[6] Mark G. Karpovsky, Krishnendu Chakrabarty, and Lev B. Levitin. On a new class of codes for identifying vertices in graphs. IEEE Trans. Inform. Theory, 44(2):599-611, 1998.

[7] Brendon Stanton. PhD thesis, Iowa State University, in progress. 